\title{
Ligustrum lucidum como uma espécie invasora oportunista em uma Floresta com Araucária no sul do Brasil
}

Ligustrum lucidum as an opportunist invasive species in an Araucaria Forest in South Brazil

\author{
Amanda da Silva Nunes ${ }^{1}$, Pedro Higuchi ${ }^{1,2}$, Ana Carolina da Silva ${ }^{1}$, Ricardo de Vargas Kilca ${ }^{1}$, \\ Mariele Alves Ferrer da Silva ${ }^{1}$ \& Janaina Gabriela Larsen ${ }^{1}$
}

\begin{abstract}
Resumo
Objetivou-se inferir sobre os mecanismos de invasão (oportunismo x competição) de Ligustrum lucidum em um fragmento de floresta com araucária em Lages, Santa Catarina. O fragmento foi inventariado nos anos de $2012 \mathrm{e}$ 2015, por meio de parcelas, onde foram avaliados os indivíduos arbóreos com DAP maior ou igual a 5,0 cm. As parcelas foram classificadas em invadidas (I) e não invadidas (NI) por L. lucidum e foram calculadas as taxas de dinâmica para a comunidade e populações. A influência de L. lucidum sobre as taxas da comunidade foi investigada por meio de regressões logísticas e o padrão de coexistência das espécies nos setores I e NI pelo índice de $c$-score. No fragmento, foram observadas maiores taxas de recrutamento do que mortalidade e maior ganho do que perda em área basal. A comunidade apresentou maior recrutamento nos locais com maior abundância de L. lucidum. Enquanto nas parcelas NI observou-se a segregação espacial das espécies, nas parcelas I as mesmas ocorreram de forma aleatória. Conclui-se que L. lucidum está aumentando sua importância relativa na comunidade, estando associado a parcelas desestruturadas em relação ao padrão de co-ocorrência das espécies, o que sugere seu caráter oportunista. Palavras-chave: espécie exótica, Floresta Atlântica, invasão biológica, padrões demográficos, Santa Catarina.
\end{abstract}

\begin{abstract}
The objective of this study was to infer the mechanisms of invasion (opportunism vs. competition) of Ligustrum lucidum in an Araucaria Forest fragment, in the municipallity of Lages, SC. This fragment was inventoried in 2012 and 2015, through plots, where tree individuals with dbh greater than or equal to $5.0 \mathrm{~cm}$ were evaluated. The plots were classified as invaded (I) or non-invaded (NI) by L. lucidum and the dynamics rates were determined for the tree community and populations. The influence of L. lucidum presence on demographic rates of community was evaluated by logistic regressions and the evaluation of species coexistence in I and NI sectors was performed by c-score index. In fragment, the recruitment rate was greater than mortality and basal area gain rate was greater than basal area loss. The community had a greater recruitment in plots with high L. lucidum abundance. While there was spatial segregation of species in NI plots, there was random pattern in I plots. We conclude that L. lucidum is increasing its relative importance in community, being associated to plots with random species co-occurrence, suggesting its opportunist nature.
\end{abstract}

Key words: exotic species, Atlantic Forest, biological invasion, demographic patterns, Santa Catarina.

\section{Introdução}

A invasão biológica de áreas naturais por espécies exóticas representa um dos principais problemas ambientais da atualidade, uma vez que suas consequências afetam negativamente a biodiversidade, o funcionamento dos ecossistemas, além de causar prejuízos econômicos (Charles \&
Dukes 2008). Neste sentido, estudos sobre processo de invasão biológica têm sido o foco de várias pesquisas nas últimas décadas, tendo como propósito caracterizar a autoecologia de espécies invasoras e identificar fatores que levam as comunidades biológicas a serem mais ou menos susceptíveis à invasão (Moles et al. 2012). Em uma ampla revisão

\footnotetext{
${ }^{1}$ Universidade do Estado de Santa Catarina, Depto. Engenharia Florestal, Av. Luiz de Camões 2090, 88520-000, Lages, SC, Brasil.

${ }^{2}$ Autor para correspondência: higuchip@gmail.com
} 
bibliográfica, Moles et al. (2012) destacaram que espécies invasoras apresentam características que representam vantagem competiva em relação às nativas, como elevada taxa de crescimento demográfico e de sobrevivência, boa resistência à herbivoria e elevada plasticidade de atributos funcionais. Moles et al. (2012) também relataram que ambientes com maior diversidade de espécies podem ser mais resistentes à invasão, pelo fato de existir nestas condições uma maior competição interespecífica. No entanto, outros padrões também podem ser observados, pois, como os mesmos autores ressaltaram, a relação entre as características da comunidade residente e a sua susceptibilidade à invasão é de elevada complexidade, tendo em vista que a mesma é condicionada por diversos fatores ecológicos (e.g., processos neutros, distúrbios e produtividade).

Quando se trata de invasão biológica, um certo cuidado é necessário quanto à questão semântica sobre o tema, considerando que nas últimas décadas o assunto foi abordado por disciplinas distintas, o que resultou na proliferação de termos, as vezes com o mesmo significado (Blackburn et al. 2011). Neste sentido, alguns estudos (Richardson et al. 2000; Blackburn et al. 2011; Moro et al. 2012) têm destacado que para uma espécie exótica ser considerada invasora, a mesma tem que ser capaz de ter superado uma série de barreiras ecológicas e se estabelescer, formar uma população autorregenerante e se dispersar para novas áreas geográficas.

Segundo MacDougall \& Turkington (2005), as espécies invasoras podem se tornar dominantes em comunidades a partir de dois caminhos: i) vantagem competitiva na exploração de recursos limitados ou, ii) oportunismo, pela menor susceptibilidade a fatores que controlam a densidade populacional de outras espécies na comunidade. Se na dominância por meio da vantagem competitiva a espécie invasora tem um papel ativo e determinante na estruturação da comunidade, na dominância por menor susceptibilidade a fatores externos a espécie ocorre como uma oportunista, tendo um papel passivo em relação a estruturação da comunidade. Considerando que, de acordo com as regras de montagem de comunidades biológicas (assembly rules), as interações competitivas resultam em padrões de co-ocorrência de espécies não-aleatórios (Gotelli \& McCabe 2002), espera-se que o papel mais ativo ou passivo da espécie invasora possa ser identificado a partir de investigações sobre como as espécies tendem a coexistirem no espaço e no tempo. Neste sentido, se, por um lado, a presença de uma invasora em um comunidade residente com padrão não-aleatório de coexistência de espécies é um forte indicativo de que a mesma seja uma boa competidora, por outro lado, espera-se que espécies oportunistas ocorram preferencialmente em comunidades com padrões aleatórios de coexistência.

Estudos que permitam inferências sobre o tipo de mecanismo dos processos de invasão são fundamentais, pois permitem a definição de estratégias de controles mais eficientes em áreas onde a invasão biológica representa um problema ambiental. No Brasil, o Instituto Hórus (2016) cita espécies do gênero Ligustrum como invasoras que têm causado diversos problemas em áreas de florestas naturais. No sul do país, nas regiões com Floresta Ombrófila Mista, se destaca Ligustrum lucidum W.T.Aiton, que já foi diagnosticado como invasor em fragmentos florestais por diversos autores (Cordeiro \& Rodrigues 2007; Sonego et al. 2007; Guidini et al. 2014). Essa espécie é nativa da Ásia, apresenta alta capacidade regenerativa e grande produção de frutos carnosos, que se tornam atrativos para a alimentação da avifauna (Instituto Hórus 2016), sendo uma espécie muito utilizada na arborização urbana (Lorenzi et al. 2003).

Considerando o exposto, a partir de investigações dos padrões demográficos da comunidade e de co-existência das espécies, o presente estudo teve como objetivo avaliar o processo de invasão por L. lucidum em uma área de Floresta Ombrófila Mista no Sul do Brasil. Para isto, verificou-se: i) se a espécie apresentou incremento populacional no período avaliado, o que seria um indicativo de que a mesma tenha superado barreiras ecológicas para o estabelecimento na área; ii) a influência da presença de L. lucidum sobre a dinâmica da comunidade avaliada; e iii) a existência de padrões aleatórios ou não-aleatórios de coexistência das espécies na comunidade residendente invadida e não invadida, com o propósito de inferir se a invasão ocorre por meio da eficiência competitiva ou oportunismo.

\section{Material e Métodos}

$\mathrm{O}$ estudo foi realizado em um fragmento florestal localizado na propriedade da Empresa de Pesquisa Agropecuária e Extensão Rural de Santa Catarina (EPAGRI), situada na área urbana do município de Lages, planalto sul-catarinense, nas coordenadas $27^{\circ} 48^{\prime} 18,40^{\prime \prime}$ S e $50^{\circ} 20^{\prime} 4,63^{\prime \prime} \mathrm{O}$, com altitude aproximada de $930 \mathrm{~m}$ (material suplementar 
em $<$ https://doi.org/10.6084/m9.figshare.4980680. v2 $>$ ). $\mathrm{O}$ fragmento possui aproximadamente 11 ha e a vegetação é classificada como Floresta Ombrófila Mista (FOM) Montana, segundo o IBGE (2012). $\mathrm{O}$ clima predominante na região, de acordo com a classificação de Köppen, é do tipo $\mathrm{Cfb}$, com ocorrência de geadas frequentes. De acordo com a estação meteorológica de Lages, administrada pela EPAGRI/CIRAM/INMET, a precipitação anual média (1970-2010) é de 1.682,80 mm, com chuvas bem distribuídas durante o ano e temperatura média anual entre 15 e $17^{\circ} \mathrm{C}$. A área de estudo está inserida na Bacia Hidrográfica do Rio Canoas e do Rio Pelotas, com topografia, em sua maior parte, suave-ondulada a ondulada.

Atualmente, o fragmento florestal se encontra protegido contra perturbações de origem antropogênicas, no entanto, segundo entrevistas realizadas com funcionários da EPAGRI, houve corte seletivo de espécies arbóreas e entrada de gado frequente entre as décadas de 1960 e 1970. Até 2008, houve a entrada eventual de gado na área. Também se destaca a utilização, até o ano de 2005, de Ligustrum lucidum como árvore ornamental na arborização do estacionamento adjacente ao fragmento, que pode ter sido uma fonte de propágulos, sendo que estes foram posteriormente removidos e substituídos por espécies arbóreas nativas.

O primeiro inventário do fragmento foi realizado em 2012 por Guidini et al. (2014). Os autores alocaram cinco transecções de $20 \mathrm{~m}$ de largura por $100 \mathrm{~m}$ de comprimento, perpendiculares à borda do fragmento, com distância mínima de 100 $m$ entre si. Cada transecção foi dividida em cinco parcelas de $20 \times 20 \mathrm{~m}$, totalizando 25 parcelas . Todos os indivíduos arbóreos com DAP (diâmetro a altura do peito, medido a $1,30 \mathrm{~m}$ do solo) maior ou igual a $5,0 \mathrm{~cm}$ que se encontravam no interior das parcelas foram identificados, quando possível, a nível de espécies, marcados com plaquetas de alumínio e tiveram sua circunferência mensurada. Três anos após a primeira avaliação, em 2015, as mesmas parcelas foram novamente inventariadas, sendo contabilizados os indivíduos mortos e sobreviventes. Os novos indivíduos que atingiam $5,0 \mathrm{~cm}$ de DAP foram incorporados ao inventário e considerados como recrutas. Os espécimes foram coletados para identificação em laboratório, com base em bibliografias especializadas e por especialistas, sendo as famílias organizadas segundo a classificação do Angiosperm Phylogeny Group (APG IV 2016).
Foi realizada a classificação das parcelas em invadidas (I) e não invadidas (NI), de acordo com a presença ou ausência de Ligustrum lucidum no primeiro inventário. A determinação da similaridade florístico-estrutural entre elas foi realizada por meio da distância de Bray-Curtis (BC), para os dois anos de avaliação. Para o fragmento como um todo, as taxas de dinâmica foram calculadas para a comunidade e para cada espécie: taxas de mortalidade, recrutamento, mudança líquida em número de indivíduos e em área basal, ganho e perda em área basal e rotatividade em número de indivíduos e em área basal. Para cada setor (I e NI), as mesmas taxas foram calculadas para as espécies com as cinco populações mais abundantes e para as comunidades dos setores invadido e não invadido. Para isso, foram utilizados os modelos algébricos baseados em Sheil \& May (1996) e Oliveira Filho et al. (2007):

$$
\begin{aligned}
& \mathrm{M}=\left[1-\left(\frac{\mathrm{N} 0 \text {-Nmort }}{\mathrm{N} 0}\right)^{1 / \mathrm{t}}\right] \times 100 \\
& \mathrm{R}=\left[1-\left(1-\frac{\mathrm{r}}{\mathrm{Nt}}\right)^{1 / \mathrm{t}}\right] \times 100 \\
& \mathrm{Ml}_{\mathrm{NI}}=\left[\left(\frac{\mathrm{Nt}}{\mathrm{No}}\right)^{1 / \mathrm{t}}-100\right] \times 100 \\
& \mathrm{M}_{\mathrm{Ab}}=\left[\left(\frac{\mathrm{Abf}}{\mathrm{Abi}}\right)^{1 / \mathrm{t}}-100\right] \times 100 \\
& \mathrm{TG}=\left[1-\left(1-\left(\frac{\text { Abganho }}{\mathrm{Abf}}\right)^{1 / \mathrm{t}}\right) \times 100\right] \\
& \mathrm{TP}=\left[1-\left(\frac{\mathrm{Abi}-\mathrm{Abperda}}{\mathrm{Abi}}\right)^{1 / \mathrm{t}}\right] \times 100 \\
& \mathrm{R}_{\mathrm{NI}}=\frac{\mathrm{M}+\mathrm{R}}{2} \\
& \mathrm{R}_{\mathrm{Ab}}=\frac{\mathrm{TG}+\mathrm{TP}}{2}
\end{aligned}
$$

em que: $\mathrm{M}=$ taxa de mortalidade anual $\left(\%\right.$.ano $\left.{ }^{-1}\right)$; N0 = número inicial de árvores; Nmort = número de árvores mortas; $t=$ intervalo de tempo entre os inventários; $\mathrm{R}=$ taxa de recrutamento anual $\left(\%\right.$.ano $\left.{ }^{-1}\right) ; \mathrm{r}=$ número de árvores recrutadas; $\mathrm{Nt}$ = número final de árvores sobreviventes depois de $\mathrm{t} ; \mathrm{Ml}_{\mathrm{NI}}=$ taxa de mudança líquida em número de indivíduos $\left(\% \cdot \mathrm{ano}^{-1}\right) ; \mathrm{Ml}_{\mathrm{Ab}}=$ taxa de mudança líquida em área basal $\left(\% \cdot \mathrm{ano}^{-1}\right) ; \mathrm{Abf}=$ área basal final $\left(\mathrm{m}^{2} / \mathrm{ha}\right) ; \mathrm{Abi}=$ área basal inicial $\left(\mathrm{m}^{2} / \mathrm{ha}\right) ; \mathrm{TG}$ $=$ taxa de ganho em área basal $\left(\% \cdot\right.$ ano $\left.^{-1}\right)$; Abganho 
= ganho em área basal a partir do crescimento dos sobreviventes e recrutamento $\left(\mathrm{m}^{2} / \mathrm{ha}\right) ; \mathrm{TP}=$ taxa de perda em área basal $\left(\%\right.$.ano $\left.{ }^{-1}\right)$; Abperda = perda em área basal a partir do decremento dos sobreviventes (por ex., perda de bifurcações do tronco) e mortalidade $\left(\mathrm{m}^{2} / \mathrm{ha}\right) ; \mathrm{R}_{\mathrm{NI}}=$ taxa de rotatividade em número de indivíduos $\left(\%\right.$.ano $\left.{ }^{-1}\right)$; $\mathrm{R}_{\mathrm{Ab}}=$ taxa de rotatividade em área basal $\left(\%\right.$. ano $\left.{ }^{-1}\right)$.

Após a verificação da normalidade dos dados por meio do teste de Shapiro-Wilk, os valores das taxas foram comparados entre as áreas invadida e não invadida por meio do teste $t$, no caso de distribuição normal, e por meio do teste de MannWhitney, no caso de distribuição não normal. Pelo fato de as taxas de mortalidade, recrutamento, ganho e perda em área basal representarem proporções, foram utilizadas regressões logísticas para se avaliar a influência da presença de Ligustrum lucidum (abundância e área basal, consideradas como variáveis independentes) sobre as mesmas. Para isto, como variáveis dependentes, foram determinadas, para cada parcela, as proporções de indivíduos da comunidade recrutados e mortos, e as proporções de área basal ganhada e perdida, que refletem exatamente as taxas demográficas avaliadas. No caso de relações significativas, a validação dos modelos se deu por meio da análise de resíduos (distribuição em função dos valores ajustados e da variável explicativa). A estruturação da comunidade foi avaliada pelo padrão de coexistência das espécies, utilizando o índice c-score, proposto por Stone \& Roberts (1990). Os valores de $c$-score observados para cada um dos setores (I e NI), relativos à invasão, foram comparados com os valores de c-score determinados a partir de um modelo nulo, construído por meio do algoritmo swap (Gotelli \&
Entsminger 2003), cuja significância foi verificada por meio de um teste de permutação $(\mathrm{n}=999)$. Valores de $c$-score significativamente maiores do que os simulados pelo modelo nulo indicam uma comunidade estruturada, com predominância de segregação das espécies nas parcelas; valores de c-score abaixo dos valores simulados indicam uma comunidade estruturada, com predominância de coexistência das espécies; valores de $c$-score entre os valores simulados evidenciam uma comunidade sem estruturação, de padrão aleatório (Gotelli \& Entsminger 2004). Todas as análises foram realizadas por meio da linguagem de programação estatística R (R Development Core Team 2016), utilizando as funções oecosimu e nestedchecker do pacote Vegan (Oksanen et al. 2016).

\section{Resultados}

Para a comunidade, o recrutamento $(2,39 \%$. $a^{-1} o^{-1}$ foi superior à mortalidade $\left(1,91 \%\right.$.ano $\left.{ }^{-1}\right)$, resultando em mudança líquida positiva no número total de indivíduos $\left(0,50 \% \cdot\right.$ ano $\left.^{-1}\right)$ e em rotatividade em número de indivíduos de $2,15 \%$. $a^{-1}$. Acompanhando esse incremento, a taxa de ganho em área basal $\left(4,21 \%\right.$.ano $\left.{ }^{-1}\right)$ foi superior à de perda $\left(2,22 \% \cdot\right.$ ano $\left.^{-1}\right)$. Assim, a taxa de mudança líquida em área basal foi de 2,07\%.ano ${ }^{-1} \mathrm{e}$ a rotatividade em área basal foi de $3,22 \%$.ano ${ }^{-1}$. Do total das 25 parcelas alocadas no fragmento, 18 encontram-se invadidas por Ligustrum lucidum (I) e sete não apresentaram invasão (NI). A análise da relação entre a presença de Ligustrum lucidum e as taxas demográficas da comunidade indicou o maior recrutamento de indivíduos de espécies arbóreas nos locais com maior abundância da espécie invasora (Tab. 1). Quando comparadas entre os setores avaliados, não foram observadas

Tabela 1 - Coeficientes angulares de regressões logísticas, com suas respectivas significâncias, para avaliação da influência da presença de Ligustrum lucidum (abundância e área basal) sobre as proporções de indivíduos mortos e recrutados, e de ganho e perda em área basal, refletindo as taxas demográficas do componente arbóreo (comunidade como um todo, incluindo exóticas) de um remanescente de Floresta Ombrófila Mista em Lages, SC.

Table 1 - Logistic regression angular coefficients with its significance for the evaluation of Ligustrum lucidum presence (abundance and basal area) on the proportions of dead and recruits individuals, and of basal area gain and loss, reflecting the tree component demographic rates (community as a whole, including exotic species) in an Araucaria Forest fragment, in the municipality of Lages, SC.

\begin{tabular}{lcccc}
\hline & Abundância de Ligustrum lucidum & $\boldsymbol{p}$ & Área basal de Ligustrum lucidum & $\boldsymbol{p}$ \\
\hline Mortalidade & $-0,006$ & 0,857 & $-1,329$ & 0,638 \\
Recrutamento & 0,056 & 0,035 & $-2,741$ & 0,297 \\
Ganho em área basal & $-0,003$ & 0,985 & $-3,613$ & 0,801 \\
Perda em área basal & $-0,034$ & 0,886 & $-6,112$ & 0,771 \\
\hline
\end{tabular}


diferenças significativas $(p>0,05)$ entre as taxas para I e NI (Tab. 2).

Na primeira avaliação (2012), foram amostrados 1.799 indivíduos arbóreos, pertencentes a 70 espécies e 34 famílias botânicas, enquanto que em 2015 observou-se 1.826 indivíduos, pertencentes a 72 espécies e 35 famílias (Tab. 3). Assim, no período avaliado, houve a entrada de Baccharis oblongifolia (Ruiz \& Pav.) Pers., Lafoensia vandelliana Cham. \& Schltdl. e Ligustrum sinense Lour. (esta última também exótica invasora), assim como da família Lythraceae, e a perda de Miconia cinerascens Miq. Dois indivíduos não foram identificados nos inventários devido à elevada altura da copa.

Destaca-se a ocorrência das espécies exóticas: Ligustrum lucidum, Ligustrum sinense, Pinus elliottii Engel. e Citrus limon (L.) Burm. Além dessas, Lafoensia vandelliana Cham. \& Schltdl., apesar de ser nativa no estado de SC, ainda não foi encontrada ocorrendo naturalmente na região, de forma que o status (nativa ou exótica) da mesma na área de estudo é incerto. Das espécies exóticas, L. lucidum ocorreu em elevada abundância (74 indivíduos em 2012, e 86 em 2015). Essa espécie representou 4,11\% dos indivíduos da comunidade arbórea em 2012 e 4,71\% em 2015, possuindo elevada mudança líquida em número de indivíduos $\left(5,14 \%\right.$.ano $\left.{ }^{-1}\right)$ e em área basal $\left(6,67 \%\right.$.ano $\left.{ }^{-1}\right)$ durante o período de avaliação.

Das cinco espécies mais abundantes nas áreas I e NI, Casearia decandra Jacq., Sebastiania commersoniana (Baill.) L.B.Sm. \& Downs e Ilex theezans Mart. ex Reissek se destacaram por apresentar elevada densidade de indivíduos nas duas condições (Tab. 2). Ocorrendo em elevada

Tabela 2 - Valores de taxas demográficas para as espécies mais abundantes na área invadida e não invadida por Ligustrum lucidum no ano de 2012, e para comunidade total de cada área*, em um fragmento de Floresta Ombrófila Mista em Lages, Santa Catarina. N0 = número de indivíduos inicial; $\mathrm{Nf}=$ número de indivíduos final; $\mathrm{M}=$ taxa de mortalidade $\left(\%\right.$.ano $\left.{ }^{-1}\right)$; $\mathrm{R}=$ taxa de recrutamento $\left(\% \cdot \mathrm{ano}^{-1}\right) ; \mathrm{Ml}_{\mathrm{NI}}=$ taxa de mudança líquida em número de indivíduos $\left(\% \cdot \mathrm{ano}^{-1}\right) ; \mathrm{R}_{\mathrm{NI}}=$ taxa de rotatividade em número de indivíduos $\left(\% \cdot \mathrm{ano}^{-1}\right) ; \mathrm{TG}=$ taxa de ganho em área basal $\left(\%\right.$.ano $\left.{ }^{-1}\right)$; $\mathrm{TP}=$ taxa de perda em área basal $\left(\% \cdot\right.$ ano $\left.^{-1}\right) ; \mathrm{Ml}_{\mathrm{Ab}}=$ taxa de mudança líquida em área basal $\left(\% \cdot \mathrm{ano}^{-1}\right) ; \mathrm{R}_{\mathrm{Ab}}=$ taxa de rotatividade em área basal $\left(\%\right.$.ano $\left.{ }^{-1}\right)$. Table 2 - Values of demographic rates for the most abundant species in Ligustrum lucidum invaded and non-invaded areas, in 2012, and for the whole community for each area*, in an Araucaria Forest fragment, in the municipality of Lages, Santa Catarina. N0 = initial number of individuals; $\mathrm{Nf}=$ final number of individuals; $\mathrm{M}=$ mortality rate $\left(\% \cdot \mathrm{year}^{-1}\right) ; \mathrm{R}=$ recruitment rate $\left(\% \cdot \mathrm{year}^{-1}\right) ; \mathrm{Ml}_{\mathrm{NI}}=$ number of individuals net change rate $\left(\% . y^{-1}{ }^{-1}\right) ; \mathrm{R}_{\mathrm{NI}}=$ number of individuals turnover rate $\left(\%\right.$.year $\left.{ }^{-1}\right) ; \mathrm{TG}=$ basal area gain rate $\left(\% . y e a r^{-1}\right) ; \mathrm{TP}=$ basal area loss rate $\left(\%\right.$.year $\left.{ }^{-1}\right) ; \mathrm{Ml}_{\mathrm{Ab}}=$ basal area net change rate $\left(\% \cdot\right.$ year $\left.^{-1}\right) ; \mathrm{R}_{\mathrm{Ab}}=$ area basal turnover rate $\left(\% \cdot \mathrm{ano}^{-1}\right)$.

\begin{tabular}{lcccccccccc}
\hline Espécies & $\mathbf{N 0}$ & $\mathbf{N f}$ & $\mathbf{M}$ & $\mathbf{R}$ & $\mathbf{M I}_{\mathbf{N I}}$ & $\mathbf{R}_{\mathbf{N I}}$ & $\mathbf{T G}$ & $\mathbf{T P}$ & $\mathbf{M I}_{\mathbf{A b}}$ & $\mathbf{R}_{\mathbf{A b}}$ \\
\hline Área invadida & & & & & & & & & & \\
Casearia decandra & 145 & 156 & 0,69 & 3,09 & 2,47 & 1,89 & 1,43 & 1,1 & 3,61 & 1,26 \\
Sebastiania commersoniana & 131 & 129 & 1,29 & 0,78 & $-0,51$ & 1,04 & 4,14 & 2,34 & 1,88 & 3,24 \\
Myrcia selloi & 91 & 88 & 3,02 & 1,93 & $-1,11$ & 2,48 & 6,36 & 3,14 & 3,44 & 4,75 \\
Ilex theezans & 82 & 87 & 1,23 & 3,16 & 1,99 & 2,2 & 5,45 & 0,83 & 4,89 & 3,14 \\
Ligustrum lucidum & 74 & 85 & 2,78 & 7,17 & 4,73 & 4,97 & 8,06 & 2,06 & 6,53 & 5,06 \\
Total - comunidade da área invadida & 1294 & 1319 & 1,78 & 2,41 & 0,64 & 2,10 & 4,07 & 2,11 & 2,03 & 3,09 \\
\hline Área não invadida & & & & & & & & & & \\
Casearia decandra & 54 & 61 & 1,25 & 5,18 & 4,15 & 3,22 & 5,18 & 0,47 & 4,97 & 2,83 \\
Jacaranda puberula & 36 & 35 & 0,93 & 0 & $-0,93$ & 0,47 & 2,71 & 0,2 & 2,58 & 1,46 \\
Sebastiania commersoniana & 33 & 33 & 1,02 & 1,02 & 0 & 1,02 & 2,81 & 1,03 & 1,83 & 1,92 \\
Cinnamodendron dinisii & 32 & 31 & 2,13 & 1,09 & $-1,05$ & 1,61 & 4,18 & 1,58 & 2,72 & 2,88 \\
Ilex theezans & 31 & 30 & 1,09 & 0 & $-1,09$ & 0,54 & 3,61 & 1,73 & 1,96 & 2,67 \\
Total - comunidade da área não invadida & 505 & 507 & 2,23 & 2,36 & 0,13 & 2,29 & 4,58 & 2,51 & 2,18 & 3,55 \\
\hline * Taxas demográficas para as comunidades invadida e não invadida não diferiram entre si $(p>0,05)$ de acordo com o teste de Mann-Whitney (taxas de \\
mortalidade, recrutamento, ganho e perda em área basal, mudança líquida em número de indivíduos e em área basal e rotatividade em número de indivíduos) e o \\
teste t (rotatividade em área basal).
\end{tabular}


Tabela 3 - Espécies amostradas no componente arbóreo de um fragmento de Floresta Ombrófila Mista invadido por Ligustrum lucidum em Lages, Santa Catarina. N0 = número de indivíduos inicial; Nf = número de indivíduos final; $\mathrm{Ml}_{\mathrm{NI}}=$ taxa de mudança líquida em número de indivíduos $\left(\% \cdot \mathrm{ano}^{-1}\right) ; \mathrm{Abi}=$ área basal inicial $\left(\mathrm{m}^{2} \cdot \mathrm{ha}^{-1}\right) ; \mathrm{Abf}=$ área basal final $\left(\mathrm{m}^{2} \cdot \mathrm{ha}^{-1}\right) ; \mathrm{Ml}_{\mathrm{Ab}}=$ taxa de mudança líquida em área basal $\left(\%\right.$.ano $\left.{ }^{-1}\right)$.

Table 3 - Sampled species in the tree component of an Araucaria Forest fragment invaded by Ligustrum lucidum in the municipality of Lages, Santa Catarina. N0 = initial number of individuals; $\mathrm{Nf}=$ final number of individuals; $\mathrm{Ml}_{\mathrm{NI}}=$ number of individuals net change rate $\left(\%\right.$.year $\left.{ }^{-1}\right) ; \mathrm{Abi}=$ initial basal area $\left(\mathrm{m}^{2} \cdot \mathrm{ha}^{-1}\right) ; \mathrm{Abf}=$ final basal area $\left(\mathrm{m}^{2} \cdot \mathrm{ha}^{-1}\right) ; \mathrm{Ml}_{\mathrm{Ab}}=$ basal area net change rate $\left(\%\right.$.year $\left.{ }^{-1}\right)$.

\begin{tabular}{|c|c|c|c|c|c|c|}
\hline Famílias/espécies & No & Nf & $\mathrm{MI}_{\mathrm{NI}}$ & Abi & Abf & $\mathbf{M l}_{\mathbf{A b}}$ \\
\hline \multicolumn{7}{|l|}{ Anacardiaceae } \\
\hline Lithraea brasiliensis Marchand & 74 & 76 & 0,89 & 1,290 & 1,393 & 2,61 \\
\hline Schinus terebinthifolius Raddi & 6 & 6 & 0,00 & 0,032 & 0,035 & 3,32 \\
\hline \multicolumn{7}{|l|}{ Annonaceae } \\
\hline Annona rugulosa (Schltdl.) H.Rainer & 3 & 3 & 0,00 & 0,046 & 0,069 & 14,36 \\
\hline \multicolumn{7}{|l|}{ Aquifoliaceae } \\
\hline Ilex dumosa Reissek & 6 & 6 & 0,00 & 0,196 & 0,214 & 3,03 \\
\hline Ilex microdonta Reissek & 7 & 7 & 0,00 & 0,081 & 0,083 & 0,95 \\
\hline Ilex paraguariensis A.St.-Hil. & 2 & 2 & 0,00 & 0,021 & 0,021 & 0,00 \\
\hline Ilex theezans Mart. ex Reissek & 113 & 117 & 1,17 & 0,971 & 1,102 & 4,29 \\
\hline \multicolumn{7}{|l|}{ Araliaceae } \\
\hline Oreopanax fulvus Marchal & 1 & 1 & 0,00 & 0,004 & 0,004 & 0,00 \\
\hline \multicolumn{7}{|l|}{ Araucariaceae } \\
\hline Araucaria angustifolia (Bertol.) Kuntze & 7 & 7 & 0,00 & 0,155 & 0,158 & 0,57 \\
\hline \multicolumn{7}{|l|}{ Asteraceae } \\
\hline Baccharis oblongifolia (Ruiz \& Pav.) Pers. & 0 & 2 & 0,00 & 0,000 & 0,010 & 0,00 \\
\hline Dasyphyllum tomentosum (Spreng.) Cabrera & 3 & 3 & 0,00 & 0,084 & 0,101 & 6,39 \\
\hline Dasyphyllum spinescens (Less.) Cabrera & 2 & 2 & 0,00 & 0,086 & 0,099 & 5,04 \\
\hline Gochnatia polymorpha (Less.) Cabrera & 66 & 62 & $-2,06$ & 1,235 & 1,264 & 0,76 \\
\hline Piptocarpha angustifolia Dusén & 1 & 1 & 0,00 & 0,018 & 0,045 & 34,82 \\
\hline Vernonanthura discolor (Spreng.) H.Rob. & 29 & 27 & $-2,35$ & 0,943 & 0,973 & 1,03 \\
\hline \multicolumn{7}{|l|}{ Bignoniaceae } \\
\hline Jacaranda puberula Cham. & 56 & 55 & $-0,60$ & 0,970 & 1,037 & 2,24 \\
\hline \multicolumn{7}{|l|}{ Canellaceae } \\
\hline Cinnamodendron dinisii Schwacke & 87 & 83 & $-1,56$ & 1,179 & 1,201 & 0,63 \\
\hline \multicolumn{7}{|l|}{ Cardiopteridaceae } \\
\hline Citronella gongonha (Mart.) R.A.Howard & 1 & 1 & 0,00 & 0,002 & 0,003 & 1,61 \\
\hline \multicolumn{7}{|l|}{ Celastraceae } \\
\hline Maytenus dasyclada Mart. & 17 & 19 & 3,78 & 0,240 & 0,253 & 1,78 \\
\hline \multicolumn{7}{|l|}{ Clethraceae } \\
\hline Clethra scabra Pers. & 39 & 39 & 0,00 & 1,159 & 1,226 & 1,90 \\
\hline \multicolumn{7}{|l|}{ Cyatheaceae } \\
\hline Alsophila setosa Kaulf. & 7 & 6 & $-5,01$ & 0,101 & 0,086 & $-5,28$ \\
\hline \multicolumn{7}{|l|}{ Dicksoniaceae } \\
\hline Dicksonia sellowiana Hook. & 40 & 36 & $-3,45$ & 1,547 & 1,325 & $-5,04$ \\
\hline
\end{tabular}




\begin{tabular}{|c|c|c|c|c|c|c|}
\hline Famílias/espécies & No & Nf & $\mathbf{M I}_{\mathrm{NI}}$ & Abi & Abf & $\mathbf{M l}_{\mathbf{A b}}$ \\
\hline \multicolumn{7}{|l|}{ Erythroxylaceae } \\
\hline Erythroxylum cuneifolium (Mart.) O.E.Schulz & 4 & 5 & 7,72 & 0,020 & 0,025 & 7,33 \\
\hline Erythroxylum deciduum A.St.-Hil. & 6 & 6 & 0,00 & 0,073 & 0,076 & 1,53 \\
\hline \multicolumn{7}{|l|}{ Escalloniaceae } \\
\hline Escallonia bifida Link \& Otto & 3 & 10 & 49,38 & 0,036 & 0,101 & 40,85 \\
\hline \multicolumn{7}{|l|}{ Euphorbiaceae } \\
\hline Sapium glandulosum (L.) Morong & 2 & 2 & 0,00 & 0,066 & 0,070 & 1,76 \\
\hline Sebastiania commersoniana (Baill.) L.B.Sm. \& Downs & 164 & 162 & $-0,41$ & 2,082 & 2,201 & 1,87 \\
\hline \multicolumn{7}{|l|}{ Fabaceae } \\
\hline Dalbergia frutescens (Vell.) Britton & 20 & 20 & 0,00 & 0,145 & 0,164 & 4,20 \\
\hline Mimosa scabrella Benth. & 11 & 9 & $-6,47$ & 0,388 & 0,350 & $-3,40$ \\
\hline Indeterminada 1 & 1 & 1 & 0,00 & 0,057 & 0,063 & 3,11 \\
\hline \multicolumn{7}{|l|}{ Lauraceae } \\
\hline Nectandra megapotamica (Spreng.) Mez & 3 & 3 & 0,00 & 0,028 & 0,032 & 4,34 \\
\hline Ocotea puberula (Rich.) Nees & 4 & 5 & 7,72 & 0,144 & 0,155 & 2,60 \\
\hline Ocotea pulchella (Nees \& Mart.) Mez & 30 & 27 & $-3,45$ & 0,928 & 0,941 & 0,48 \\
\hline \multicolumn{7}{|l|}{ Lythraceae } \\
\hline Lafoensia vandelliana Cham. \& Schltdl. & 0 & 1 & - & 0,000 & 0,002 & - \\
\hline \multicolumn{7}{|l|}{ Melastomataceae } \\
\hline Miconia cinerascens Miq. & 1 & 0 & -100 & 0,007 & 0,000 & -100 \\
\hline Miconia ramboi Brade & 2 & 2 & 0,00 & 0,012 & 0,017 & 11,52 \\
\hline \multicolumn{7}{|l|}{ Myrtaceae } \\
\hline Acca sellowiana (O.Berg) Burret & 1 & 1 & 0,00 & 0,004 & 0,009 & 37,88 \\
\hline Blepharocalyx salicifolius (Kunth) O.Berg & 2 & 2 & 0,00 & 0,014 & 0,016 & 3,79 \\
\hline Calyptranthes concinna DC. & 9 & 9 & 0,00 & 0,046 & 0,052 & 4,32 \\
\hline Campomanesia xanthocarpa O.Berg & 1 & 1 & 0,00 & 0,004 & 0,004 & 1,90 \\
\hline Eugenia pluriflora DC. & 5 & 5 & 0,00 & 0,058 & 0,063 & 3,12 \\
\hline Eugenia pyriformis Cambess. & 5 & 5 & 0,00 & 0,046 & 0,055 & 3,13 \\
\hline Myrceugenia euosma (O.Berg) D.Legrand & 72 & 77 & 2,26 & 0,442 & 0,463 & 1,60 \\
\hline Myrcia guianensis (Aubl.) DC. & 27 & 26 & $-1,25$ & 0,375 & 0,385 & 0,86 \\
\hline Myrcia selloi (Spreng.) N. Silveira & 118 & 115 & $-0,85$ & 0,636 & 0,700 & 3,23 \\
\hline Myrcia palustris DC. & 49 & 50 & 0,68 & 0,547 & 0,583 & 2,18 \\
\hline Myrcia splendens (Sw.) DC. & 60 & 59 & $-0,56$ & 1,125 & 1,175 & 1,48 \\
\hline Myrciaria delicatula (DC.) O.Berg & 12 & 13 & 2,70 & 0,035 & 0,044 & 7,62 \\
\hline Siphoneugena reitzii D.Legrand & 2 & 2 & 0,00 & 0,007 & 0,008 & 8,55 \\
\hline \multicolumn{7}{|l|}{ Oleaceae } \\
\hline Ligustrum lucidum W.T. Aiton. & 74 & 86 & 5,14 & 0,983 & 1,194 & 6,67 \\
\hline Ligustrum sinense Lour. & 0 & 1 & - & 0,000 & 0,003 & - \\
\hline \multicolumn{7}{|l|}{ Pinaceae } \\
\hline Pinus elliottii Engel. & 1 & 1 & 0,00 & 0,002 & 0,016 & 99,25 \\
\hline
\end{tabular}




\begin{tabular}{|c|c|c|c|c|c|c|}
\hline Famílias/espécies & No & $\mathbf{N f}$ & $\mathbf{M I}_{\mathrm{NI}}$ & Abi & Abf & $\mathbf{M l}_{\mathbf{A b}}$ \\
\hline \multicolumn{7}{|l|}{ Podocarpaceae } \\
\hline Podocarpus lambertii Klotzsch ex Endl. & 47 & 48 & 0,70 & 0,634 & 0,789 & 7,57 \\
\hline \multicolumn{7}{|l|}{ Primulaceae } \\
\hline Myrsine coriacea (Sw.) R.Br. ex Roem. \& Schult. & 34 & 35 & 0,97 & 0,446 & 0,470 & 1,72 \\
\hline Myrsine lorentziana (Mez) Arechav. & 21 & 18 & $-5,01$ & 0,152 & 0,108 & $-10,81$ \\
\hline Myrsine umbellata Mart. & 56 & 60 & 2,33 & 0,446 & 0,540 & 6,53 \\
\hline \multicolumn{7}{|l|}{ Quillajaceae } \\
\hline Quillaja brasiliensis (A.St.-Hil. \& Tul.) Mart. & 3 & 3 & 0,00 & 0,071 & 0,076 & 2,63 \\
\hline \multicolumn{7}{|l|}{ Rhamnaceae } \\
\hline Rhamnus sphaerosperma $\mathrm{Sw}$. & 3 & 3 & 0,00 & 0,035 & 0,037 & 1,93 \\
\hline \multicolumn{7}{|l|}{ Rosaceae } \\
\hline Prunus myrtifolia (L.) Urb. & 11 & 11 & 0,00 & 0,246 & 0,270 & 3,24 \\
\hline \multicolumn{7}{|l|}{ Rutaceae } \\
\hline Citrus limon (L.) Burm & 1 & 1 & 0,00 & 0,005 & 0,006 & 4,06 \\
\hline Zanthoxylum kleinii (R.S.Cowan) P.G.Waterman & 47 & 46 & $-0,71$ & 0,919 & 0,991 & 2,55 \\
\hline Zanthoxylum rhoifolium Lam. & 19 & 20 & 1,72 & 0,193 & 0,224 & 5,10 \\
\hline \multicolumn{7}{|l|}{ Salicaceae } \\
\hline Casearia decandra Jacq. & 199 & 217 & 2,93 & 1,450 & 1,633 & 4,03 \\
\hline Casearia obliqua Spreng. & 15 & 14 & $-2,27$ & 0,248 & 0,267 & 2,54 \\
\hline Xylosma ciliatifolia (Clos) Eichler & 5 & 4 & $-7,17$ & 0,020 & 0,019 & $-1,47$ \\
\hline Xylosma tweediana (Clos) Eichler & 2 & 2 & 0,00 & 0,007 & 0,007 & $-0,56$ \\
\hline \multicolumn{7}{|l|}{ Sapindaceae } \\
\hline Allophylus edulis (A.St.-Hil. et al.) Hieron. ex Niederl. & 6 & 7 & 5,27 & 0,077 & 0,089 & 4,77 \\
\hline Matayba elaeagnoides Radlk. & 24 & 23 & $-1,41$ & 0,876 & 0,919 & 1,63 \\
\hline \multicolumn{7}{|l|}{ Solanaceae } \\
\hline Solanum mauritianum Scop. & 1 & 2 & 25,99 & 0,003 & 0,007 & 34,65 \\
\hline Solanum compressum L.B. Sm. \& Downs & 1 & 1 & 0,00 & 0,009 & 0,011 & 7,49 \\
\hline \multicolumn{7}{|l|}{ Stryraceae } \\
\hline Styrax leprosus Hook. \& Arn. & 6 & 6 & 0,00 & 0,054 & 0,061 & 4,30 \\
\hline \multicolumn{7}{|l|}{ Symplocaceae } \\
\hline Symplocos uniflora (Pohl) Benth. & 25 & 23 & $-2,74$ & 0,160 & 0,178 & 3,48 \\
\hline \multicolumn{7}{|l|}{ Winteraceae } \\
\hline Drimys brasiliensis Miers & 15 & 13 & $-4,66$ & 0,334 & 0,263 & $-7,62$ \\
\hline N.I. & 2 & 2 & 0 & 0,043 & 0,055 & 9,07 \\
\hline Total & 1799 & 1826 & 0,50 & 25,096 & 26,688 & 2,07 \\
\hline
\end{tabular}

densidade somente nas áreas NI, destacaram-se Jacaranda puberula Cham. e Cinnamodendron diniisii Schwacke, e somente nas áreas I, Myrcia selloi, sendo que L. lucidum ocupou a quinta colocação em densidade nessa última área. A similaridade florístico-estrutural entre os setores
I e NI se manteve a mesma para os dois anos da avaliação (BC = 0,49 para 2012 e 2015).

Avaliando as taxas demográficas das espécies de maior densidade na área I, destaca-se Ligustrum lucidum com maior recrutamento $\left(7,17 \%\right.$. ano $\left.{ }^{-1}\right) \mathrm{e}$ ganho em área basal $\left(8,06 \%\right.$.ano $\left.{ }^{-1}\right)$, o que refletiu 
nas maiores mudanças líquidas $\left(\mathrm{Ml}_{\mathrm{NI}}=4,73 \%\right.$. ano $^{-1}$ e $\mathrm{Ml}_{\mathrm{Ab}}=6,53 \%$.ano $\left.{ }^{-1}\right)$ e rotatividades $\left(\mathrm{R}_{\mathrm{NI}}=\right.$ $4,97 \%$. ano ${ }^{-1}$ e $\mathrm{R}_{\mathrm{Ab}}=5,06 \%$. ano ${ }^{-1}$ ) observadas nesse grupo. Entre as espécies de maior densidade na área NI, destaca-se Casearia decandra, com elevado recrutamento $\left(5,18 \%\right.$.ano $\left.{ }^{-1}\right)$, ganho em área basal $\left(5,18 \% \cdot\right.$ ano $\left.^{-1}\right)$, mudanças líquidas $\left(\mathrm{Ml}_{\mathrm{NI}}=4,15 \%\right.$. ano $^{-1}$ e $\mathrm{Ml}_{\mathrm{Ab}}=4,97 \%$.ano $\left.{ }^{-1}\right)$ e rotatividades $\left(\mathrm{R}_{\mathrm{NI}}=\right.$ $3,22 \%$.ano ${ }^{-1}$ e $\mathrm{R}_{\mathrm{Ab}}=2,83 \%$.ano ${ }^{-1}$ ). No entanto, quando se observa o padrão de Casearia decandra na área I, observa-se que a mesma apresentou, relativamente, um menor recrutamento $(3,09 \%$. ano $\left.^{-1}\right)$, menor ganho em área basal $\left(1,43 \%\right.$. ano $\left.{ }^{-1}\right)$, menores mudanças líquidas $\left(\mathrm{Ml}_{\mathrm{NI}}=2,47 \%\right.$. ano ${ }^{-1} \mathrm{e}$ $\mathrm{Ml}_{\mathrm{Ab}}=3,61 \% \cdot$ ano $\left.^{-1}\right)$ e menor rotatividades $\left(\mathrm{R}_{\mathrm{NI}}=\right.$ $1,89 \%$.ano ${ }^{-1}$ e $\mathrm{R}_{\mathrm{Ab}}=1,26 \%$.ano ${ }^{-1}$ ) do que na área NI. Da mesma forma, Sebastiania commersoniana apresentou, relativamente, maior mortalidade e menor recrutamento na área invadida no que na área não invadida.

$\mathrm{O}$ índice de $c$-score indicou padrões distintos para as áreas invadidas e não invadidas (Tab. 4). Para áreas invadidas, o padrão foi aleatório para ambos os períodos avaliados (2012 e 2015), indicando comunidade não estruturada. Para a área não invadida, o padrão foi de segregação das espécies para ambos os períodos.

\section{Discussão}

No perfil florístico da comunidade residente, além de Ligustrum lucidum, observou-se outras três espécies exóticas (L. sinense, Pinus elliottii e Citrus limon) que possuem potencial invasor conforme indicado pelo Instituto Hórus (2016). Estas, apesar de ocorrerem em baixa densidade (1 ind.ha ${ }^{-1}$ ), devem ser monitoradas, uma vez que podem se tornar abundantes no futuro. Também, outra espécie ainda não citada como de ocorrência natural na região foi encontrada, provavelmente devido ao plantio dessa na arborização próximo à área de estudo - estas poderia ter se dispersado para a área de floresta - ou pode ter havido, no passado, um eventual plantio das mudas na floresta.

De forma geral, a dinâmica do componente arbóreo foi caracterizada por estar em um processo de estruturação, com ganho em número de indivíduos e em área basal, e estabilidade na riqueza, com ganho de apenas duas espécies. Esse padrão é comum em florestas em fase mais tardia do processo de recuperação pós-distúrbio, conforme foi observado também por Formento et al. (2004) e Salami et al. (2014). Conhecendo o histórico da área, confirma-se esse processo, pois esta foi impactada por cortes seletivos de espécies arbóreas madeireiras e pela entrada de gado no passado, da mesma forma que outras florestas situadas na mesma bacia hidrográfica (Sevegnani et al. 2012). Quanto às pequenas mudanças observadas na riqueza de espécies, estas são comuns em um curto período de avaliação (Nunes et al. 2016) e estudos de longo prazo são indicados para inferências mais conclusivas sobre uma alteração futura na riqueza.

O maior recrutamento de espécies arbóreas nos locais com a maior quantidade de Ligustrum lucidum sugere que, na área, os mesmos fatores que favorecem o recrutamento de espécies nativas, também favorecem o estabelecimento desta espécie invasora. Este aspecto sugere um comportamento oportunista no fragmento estudado, com a possibilidade de que L. lucidum esteja assumindo função semelhante às nativas no processo de sucessão e recuperação pós-distúrbio na área. Diante desta inferência, deve-se considerar que, i) assim como as demais espécies da comunidade residente, $L$. lucidum está colonizando áreas

Tabela 4 - Índice de c-score do componente arbóreo para o setor invadido e não invadido por Ligustrum lucidum, em uma área de Floresta Ombrófila Mista em Lages, Santa Catarina.

Table 4 - C-score index of tree component in Ligustrum lucidum invaded and non-invaded sectors, in an Araucaria Forest area, in the municipality of Lages, Santa Catarina.

\begin{tabular}{lcccc}
\hline Setores & \multicolumn{4}{c}{$c$-score } \\
\hline & Observado & Média (simulado) & Erro padrão (simulado) & $p$ \\
Invadido - 2012 & 5,57 & 5,54 & 0,0009 & 0,323 \\
Invadido - 2015 & 5,66 & 5,62 & 0,0009 & 0,305 \\
Não invadido - 2012 & 0,63 & 0,61 & 0,0002 & 0,003 \\
Não invadido - 2015 & 0,73 & 0,69 & 0,0002 & 0,001 \\
\hline
\end{tabular}


que podem ser ambientalmente favoráveis ao seu recrutamento e ii) tanto L. lucidum, quanto as espécies nativas, podem estar atuando como facilitadores no estabelecimento uma das outras. Além disso, deve ser considerado o nível de inclusão amostral utilizado (DAP maior ou igual a $5 \mathrm{~cm}$ ), de forma que, se outras fases ontogenéticas fossem avaliadas, resultados distintos poderiam ser encontrados.

Enquanto na área não invadida, Casearia decandra, espécie muito comum no sub-bosque da Floresta Ombrófila Mista (Higuchi et al. 2012), se destacou por ser a de maior incremento em abundância e dominância, indicando aumento de sua importância relativa ao longo do tempo, na área invadida, Ligustrum lucidum se destacou pelo mesmo motivo, apresentando um comportamento análogo. Assim, pode-se dizer que, no fragmento florestal, L. lucidum está conseguindo ultrapassar as barreiras ou filtros ambientais envolvidos no processo de invasão, como, por exemplo, dispersão, condições bióticas e abióticas (Theoharides \& Dukes 2007). A tendência de algumas espécies em reduzir seus desempenhos na área invadida (e.g., Casearia decandra e Sebastiania commersoniana) é um indicativo de que Ligustrum lucidum possa assumir um comportamento mais competitivo a longo tempo, o que requer um monitoramento em um prazo maior para esta comprovação.

Segundo MacDougall \& Turkington (2005), enquanto a dominância de uma espécie por superioridade competitiva ocorre em ambientes onde a competição inter-específica é intensa, tendo a espécie invasora um papel ativo na organização da comunidade (driver), no caso de dominância por oportunismo a interação entre as espécies é menor, com a invasora tendo um papel mais passivo (passenger). Assim, a partir dos resultados obtidos pela análise de c-score, é possível inferir que Ligustrum lucidum seja uma espécie oportunista no local de estudo, reforçando o exposto anteriormente quando avaliada a relação entre a presença da espécie invasora e as taxas demográficas, pois as parcelas em que esta ocorre apresentam padrão aleatório no que se refere a coexistência ou segregação de espécies, sugerindo ausência de competição intensa entre as mesmas. O fato de não ocorrer maior mortalidade e perda em área basal em função da presença de L. lucidum e os valores de similaridade florístico-estrutural terem se mantido constante nos anos avaliados, corrobora com a ideia de que o mesmo não seja o causador da desestruturação na comunidade residente, mas sim um oportunista de perturbações e distúrbios prévios. Este mesmo resultado foi observado para outras espécies arbóreas invasoras em Santa Catarina, como Hovenia dulcis Thunb. (Lazzarin et al. 2015), indicando que este é um padrão comum. Estes resultados também estão de acordo com a conclusão de Levine et al. (2004) que, a partir de uma meta-análise de trabalhos realizados com diferentes formas de vida e em várias regiões biogeográficas do planeta, inferiu que comunidades com interações inter-específicas mais intensas são mais eficientes em controlar a densidade populacional de espécies invasoras. Assim, considerando que no presente estudo a ocorrência de L. lucidum está associada a ambientes onde as espécies ocorrem de forma completamente aleatória nas parcelas, de acordo com o modelo nulo testado, sugere-se que, na área avaliada, os eventos estocásticos (e.g., dispersão de propágulos, distúrbios) tenham sido determinantes no processo de invasão por espécies arbóreas.

Conclui-se que: i) por meio da avaliação da dinâmica da floresta estudada, pode-se inferir que essa se encontra em fase de recuperação estrutural pós-distúrbio, com maiores taxas de recrutamento do que de mortalidade e maior ganho que perda em área basal; ii) locais com maior abundância de Ligustrum lucidum apresentaram maior taxa de recrutamento de espécies arbóreas, sugerindo que os mesmos fatores que favorecem o estabelecimento de espécies nativas, também favorecem a espécie invasora; iii) L. lucidum apresentou aumento de sua representatividade na floresta, ocorrendo em ambientes com um padrão aleatório em relação à coexistência e segregação das espécies. Este padrão indica que essa espécie invasora apresenta um comportamento oportunista na área de estudo, ocorrendo em locais com baixa competição inter-específica. Ainda, sugere-se que a ocorrência de eventos estocásticos, associados com a ocorrência de perturbações (destruturação da comunidade) e a chegada de propágulos, foram determinantes no processo de invasão pela espécie avaliada. Assim, é possível inferir que perturbações naturais ou antrópicas, que resultem na redução de espécies e indivíduos arbóreos, podem ser considerados como catalisadores do processo de invasão. Ressalta-se a natureza local do presente estudo, de forma que seus resultados não devem ser generalizados para a espécie invasora em questão e nem para outras áreas. Para generalizações, será necessária uma abordagem em maior escala espacial e temporal. 


\section{Agradecimentos}

À Empresa de Pesquisa Agropecuária e Extensão Rural de Santa Catarina (EPAGRI), a permissão e apoio para realizar o trabalho de campo em área de sua propriedade; à FAPESC, a concessão da bolsa de Mestrado à primeira autora e o apoio financeiro via PAP/UDESC; e ao CNPq, a concessão de bolsa de Produtividade para o segundo e o terceiro autores.

\section{Referências}

APG IV - The Angiosperm Phylogeny Group (2016) An update of the Angiosperm Phylogeny Group classification for the orders and families of flowering plants: APG IV. Botanical Journal of the Linnean Society 181: 1-20.

Blackburn TM, Pyšek P, Bacher S, Carlton JT, Duncan RP, Jarošík V, Wilson JRU \& Richardson DM (2011) A proposed unified framework for biological invasions. Trends in Ecology and Evolution 26: 333-339.

Charles H \& Dukes JS (2008) Impacts of invasive species on ecosystem services. Biological Invasions 193: 217-237.

Cordeiro J \& Rodrigues WA (2007) Caracterização fitossociológica de um remanescente de Floresta Ombrófila Mista em Guarapuava, PR. Revista Árvore 31: 545-554.

Formento S, Schorn LA \& Ramos RA (2004) Dinâmica estrutural arbórea de uma Floresta Ombrófila Mista em Campo Belo do Sul. Cerne 10: 196-212.

Gotelli NJ \& McCabe DJ (2002) Species co-occurrence: a meta-analysis of JM Diamond's Assembly rules model. Ecology 83: 2091-2096.

Gotelli NJ \& Entsminger GL (2004) EcoSim: null models software for ecology. Version 7. Acquired Intelligence Inc. e Kesey-Bear. Jericho, VT 05465. Disponível em $<$ http://garyentsminger.com/ ecosim/ index.htm>. Acesso em 25 setembro 2016.

Guidini AL, Silva AC, Higuchi P, Dalla Rosa A, Spiazzi FR, Negrini M, Ferreira TS, Salami B, Marcon AK \& Buzzi Junior F (2014) Invasão por espécies arbóreas exóticas em remanescentes florestais no Planalto Sul Catarinense. Revista Árvore 38: 469-478.

Higuchi P, Silva AC, Ferreira TS, Souza ST, Gomes JP, Silva KM, Santos KF, Linke C \& Paulino PS (2012) Influência de variáveis ambientais sobre o padrão estrutural e florístico do componente arbóreo, em um fragmento de Floresta Ombrófila Mista Montana em Lages, SC. Ciência Florestal 22: 79-90.

IBGE - Instituto Brasileiro de Geografia e Estatística (2012) Manual técnico da vegetação brasileira. Fundação Instituto Brasileiro de Geografia e Estatística, Rio de Janeiro. 271p.

Instituto Hórus (2016) Instituto Hórus de desenvolvimento e conservação ambiental. Disponível em <http:// www.institutohorus.org.br $>$. Acesso em 25 setembro 2016.

Lazzarin LC, Silva AC, Higuchi P, Souza K, Perin JE \& Cruz AP (2015) Invasão biológica por Hovenia dulcis Thunb. em fragmentos florestais na região do Alto Uruguai, Brasil. Revista Árvore 39: $1007-$ 1017.

Levine JM, Adler PB \& Yelenik SG (2004) A metaanalysis of biotic resistance to exotic plant invasions. Ecology 7: 975-989.

Lorenzi H, Souza HM, Torres MAV \& Bacher LB (2003) Árvores exóticas no Brasil: madeireiras, ornamentais e aromáticas. Plantarum, Nova Odessa. 368 .

MacDougall AS \& Turkington R (2005) Are invasive species the drivers or passengers of change in degraded ecosystems? Ecology 86: 42-55.

Moles AT, Flores-Moreno H, Bonser SP, Warton DI, Helm A, Warman L, Eldridge DJ, Jurado E, Hemmings FA, Reich PB, Caverder-Bares J, Seabloom EW, Mayfield MM, Sheil D, Djietror JC, Peri PL, Enrico L, Cabido MR, Setterfield SA, Lehmann CER \& Thomson FJ (2012) Invasions: the trail behind, the path ahead, and a test of a disturbing idea. Journal of Ecology 100: 116-127.

Moro MF, Souza VC, Oliveira-Filho AT, Queiroz LP, Fraga CN, Rodas MJN, Araújo FS \& Martins FR (2012) Alienígenas na sala: o que fazer com espécies exóticas em trabalhos de taxonomia, florística e fitossociologia? Acta Botanica Brasilica 26: 991-999.

Nunes MH, Higuchi P, Silva AC, van den Berg E \& Terra MCNS (2016) Dinâmica de populações de espécies arbóreas em fragmentos de floresta aluvial no sul de minas gerais, Brasil. Floresta 46: 57-66.

Oksanen J, Blanchet FG, Kindt R, Legendre P, Minchin PR, O'Hara RB, Simpson GL, Solymos P, Stevens MHH \& Wagner H (2016) Vegan: community ecology package. Disponível em <http://cran.rproject.org $/$ package $=$ vegan $>$. Acesso em 25 setembro 2016.

Oliveira Filho AT, Carvalho WAC, Machado ELM, Higuchi P, Appolinário V, Castro GC, Silva AC, Santos RM, Borges LF, Corrêa BS \& Alves JM (2007) Dinâmica da comunidade e populações arbóreas da borda e interior de um remanescente florestal na Serra da Mantiqueira, Minas Gerais, em um intervalo de cinco anos (1999-2004). Revista Brasileira de Botânica 30: 149-161.

R Development Core Team (2016) R: a language and environment for statistical computing. Vienna: $\mathrm{R}$ foundation for statistical computing. Disponível em $<$ http://www.Rproject.org $>$. Acesso em 25 setembro 2016.

Salami B, Higuchi P, Silva AC, Ferreira TS, Marcon AK, Buzzi Junior F \& Bento MA (2014) Influência de variáveis ambientais na dinâmica do componente 
arbóreo em um fragmento de Floresta Ombrófila Mista em Lages, SC. Scientia Forestalis 42: $197-$ 207.

Sevegnani L, da Silva TC, Gasper AL, Meyer L \& Verdi M (2012) Flora arbórea e o impacto humano nos fragmentos florestais na bacia do Rio Pelotas, Santa Catarina, Brasil. Revista de Estudos Ambientais 14: $60-73$.

Sheil D \& May RM (1996) Mortality and recruitment rate evaluations in heterogeneous tropical forests. Journal of Ecology 84: 91-100.

Sonego RC, Backes A \& Souza AF (2007) Descrição da estrutura de uma Floresta Ombrófila Mista, RS,
Brasil, utilizando estimadores não-parámetricos de riqueza e rarefação de amostras. Acta Botanica Brasilica 21: 943-955.

Stone L \& Roberts A (1990) The checkerboard score and species distributions. Oecologia 85: 74-79.

Richardson DM, Pyšek P, Rejmánek M, Barbour MG, Panetta FD \& West CJ (2000) Naturalization and invasion of alien plants: concepts and definitions. Diversity and Distribution 6: 93-107.

Theoharides KA \& Dukes JS (2007) Plant invasion across space and time: factors affecting non indigenous species success during four stages of invasion. New Phytoligist 176: 256-273.

Editor de área: Dr. Marcelo Moro Artigo recebido em 09/10/2016. Aceito para publicação em 15/08/2017. article distributed under the terms of the Creative Commons Attribution License. 